\title{
Anti-hyperalgesic effect of electroacupuncture in a model of post-incisional pain in rats
}

R. O liveira and W.A. Prado
Departamento de Farmacologia, Faculdade de Medicina de Ribeirão Preto, Universidade de São Paulo, Ribeirão Preto, SP, Brasil

\section{Correspondence \\ W.A. Prado \\ Departamento de Farmacologia \\ FM RP, USP \\ Av. Bandeirantes, 3900 \\ 14049-900 Ribeirão Preto, SP \\ Brasil \\ Fax: + 55-16-633-2301 \\ E-mail: wadprado@fmrp.usp.br \\ Publication supported by FAPESP.}

Received December 10, 1999

Accepted March 29, 2000

\section{Abstract}

Electroacupuncture has been proposed to be a low cost and practical method that allows effective pain management with minimal collateral effects. In this study we have examined the effect of electroacupuncture against the hyperalgesia developed in a model of postincisional pain in rats. A $1-\mathrm{cm}$ longitudinal incision was made through the skin and fascia of the plantar region of the animal hind paw. Mechanical hyperalgesia in the incision was evaluated $135 \mathrm{~min}$ after the surgery with von Frey filaments. The tension threshold was reduced from $75 \mathrm{~g}$ (upper limit of the test) to $1.36 \pm 0.36 \mathrm{~g}$ (mean \pm SEM) in control rats. It is shown that a 15 -min period of electroacupuncture applied $120 \mathrm{~min}$ after surgery to the Zusanli (ST36) and Sanyinjiao (SP6) points, but not to non-acupoints, produces a significant and long-lasting reduction of the mechanical hyperalgesia induced by the surgical incision of the plantar surface of the ipsilateral hind paw. The tension threshold was reduced from 75 to $27.6 \pm 4.2 \mathrm{~g}$ in animals soon after the end of electroacupuncture. The mechanical threshold in this group was about $64 \%$ less than in control. Electroacupuncture was ineffective in rats treated 10 min earlier with naloxone ( $1 \mathrm{mg} / \mathrm{kg}$, ip), thus confirming the involvement of opioid mechanisms in the antinociceptive effects of such procedure. The results indicate that post-incisional pain is a useful model for studying the antihyperalgesic properties of electroacupuncture in laboratory animals.

\section{Key words}

- Electroacupuncture

- Post-incisional pain

- Hyperalgesia

- Naloxone
Post-surgical pain is a very common type of acute pain. Its adequate management reduces morbidity and postoperative mortality. Opioids, non-steroidal antiinflammatory drugs, local anesthetics or sedatives are usually recommended for the management of post-surgical pain, but they frequently produce unsatisfactory analgesia accompanied by adverse effects. Electroacupuncture (EA) has been proposed as a low cost and practical method that can allow effective pain management with minimal side effects (1-6). Several studies have indicated that the analgesia evoked by EA depends on the participation of endogenous opioid mechanisms since the effect is inhibited by the non-selective opioid antagonist naloxone (7-9). High frequency EA produces analgesia that is partially reversed by previous reduction of the synthesis of 5-hydroxytryptamine in the central ner- 
vous system. In addition, low frequency, but not high frequency EA-induced analgesia is inhibited by naloxone $(10,11)$, thus indicating that non-opioid mechanisms may also participate in the phenomenon.

Several methods have been introduced for the study of hyperalgesic states in laboratory animals, but most of them are not suitable for the study of post-surgical pain. More recently, a model of post-incisional pain in rats was proposed to produce a hyperalgesic state that resembles the post-surgical pain (12). In the present study, we have determined whether this method can be used to evaluate EA-induced antinociception, as well as to determine if the effect is sensitive to naloxone.

We used adult male Wistar rats (150-170 g) individually housed in cages with water and food available ad libitum, under controlled light and temperature conditions (12$\mathrm{h}$ light-dark cycle, with lights off at 7:00 h). All tests were carried out in the morning. The study was conducted in accordance with the IASP guidelines on the use of laboratory animals.

Each animal was anesthetized with ether via a nose cone. The plantar aspect of the right hind paw was sterilized with a $10 \%$ povidone-iodine solution. A 1-cm longitudinal incision was made with a surgical blade through the skin and fascia of the plantar region, starting $0.5 \mathrm{~cm}$ from the proximal edge of the heel. The plantaris muscle was elevated, but its origin and insertion were left intact. After hemostasis, the skin was stitched with two 5-0 nylon sutures. After these procedures, the animal was allowed to recover in the home cage for a period of $2 \mathrm{~h}$. The rats were then lightly anesthetized with halothane via a nose cone and stimulated at the acupoints of Zusanli (ST36) and Sanyinjiao (SP6) on the right hind paw (7). The Zusanli point was located by palpation laterally to the distal end of the cranial tuberosity of the tibia. An acupuncture needle was then introduced into the space between the fibula and tibia, passing through the skin, the subcutaneous tissue and the tibial muscle. Another needle was located in the space between the Achilles tendon and the distal part of the tibia at the Sanyinjiao point. The needles were connected to the outputs of a constant current stimulator (GEA 878, KLD Biosistemas, São Carlos, SP, Brazil), and stimulated continuously for $15 \mathrm{~min}$ through the needles at the frequency of $4 \mathrm{~Hz}$. The current threshold was the lowest intensity that produced hind limb muscle contraction.

The animals were assigned to 5 experimental groups. Animals in group EA $(\mathrm{N}=9)$ received an intraperitoneal injection of saline $(0.1 \mathrm{ml} / \mathrm{kg})$, whereas those in the EANAL group $(\mathrm{N}=7)$ received an intraperitoneal injection of naloxone $(1 \mathrm{mg} / \mathrm{kg}) 10 \mathrm{~min}$ before stimulation. Group S included 8 rats that received electroacupuncture applied to non-acupuncture points in the operated paw. In these animals one needle was positioned 1 $\mathrm{mm}$ under the tibia tuberosity and the other was placed $0.5 \mathrm{~cm}$ laterally to SP6. Group SNAL $(\mathrm{N}=7)$ received an intraperitoneal injection of saline $(0.1 \mathrm{ml} / \mathrm{kg})$. The control group consisted of 10 rats. The control and S-NAL groups were sedated but not stimulated.

The mechanical threshold was measured with von Frey filaments (Stoelting, Wood Dale, IL, USA). Rats were placed in an elevated clear plastic cage with a nylon mesh bottom, which allowed easy access to the paw plantar surface. Before the EA procedures, the animals were left in the cage for approximately $15 \mathrm{~min}$ to allow behavioral accommodation. The area tested was the mid-plantar right hind paw and the mechanical thresholds were determined 1, 5, 10, 15, 20 and 30 min after EA procedures. The paw was touched with one of a series of 9 von Frey filaments with logarithmically incremental stiffness ( 0.69 to $75.858 \mathrm{~g}$, lower and upper limit of the test, respectively). Each filament was vertically applied to the plantar surface from underneath the nylon mesh floor 
through the mesh with sufficient force to bend the filament a little. A single trial consisted of 6 applications of a particular filament, applied once every 3-4 s. Testing was initiated with the $3.63 \mathrm{~g}$ filament in the middle of the series. A response was defined as a withdrawal of the stimulated paw. In the absence of a response to a particular filament, the next stronger filament was utilized; in the case of a response, the next weaker filament was presented. The up-down method was used for threshold recording (13).

The results were plotted as medians and confidence limits (95\%) and are presented in graphs as log of mean \pm SEM. The experimental groups were compared by the nonparametric Kruskal-Wallis test and differences among the data at each moment were compared by the Mann-Whitney test. The level of significance was set at $\mathrm{P}<0.05$.

Forty-one rats were utilized in the study and the results are illustrated in Figure 1. Control rats had typical hyperalgesia to mechanical stimulation as reported elsewhere (12). The tension threshold was reduced from $75 \mathrm{~g}$ (upper limit of the test) to $1.36 \pm 0.36 \mathrm{~g}$ (mean $\pm \mathrm{SD}$ ) in control rats. The EA applied to the E36 and BP6 points produced a longlasting anti-hyperalgesic effect, the mechanical thresholds remaining significantly above the control throughout the experiment. The mechanical threshold was reduced from 75 to $27.6 \pm 4.2 \mathrm{~g}$ soon after the end of electroacupuncture. The EA at points ST36 and SP6 was ineffective in animals pretreated with naloxone $(1 \mathrm{mg} / \mathrm{kg}$, ip). Thus, the pattern of EA-induced analgesia employed here depends at least in part on the activation of endogenous opioid mechanisms. The thresholds of animals stimulated at non-acupuncture points did not differ significantly from control, showing that nonspecific effects of stimulation, such as stress, are not the reason for the anti-hyperalgesic effect of the EA. All rats in the experiment were lightly anesthetized during the EA procedures, but only rats from the EA group had a significant reduction of post-incisional pain. Thus, the effectiveness of the EA is unlikely to be due to any residual anesthesia. Animals previously treated with naloxone $(1 \mathrm{mg} / \mathrm{kg}$, ip $)$ had post-incisional hyperalgesia that did not differ significantly from the control. These results indicate that the model of postincisional pain is suitable for the detection of the analgesic effect induced by EA.

Several studies indicate that activation of descending pathways in the central nervous system may be involved in the analgesia evoked by EA. Activation of the central serotonergic, cholinergic, noradrenergic and

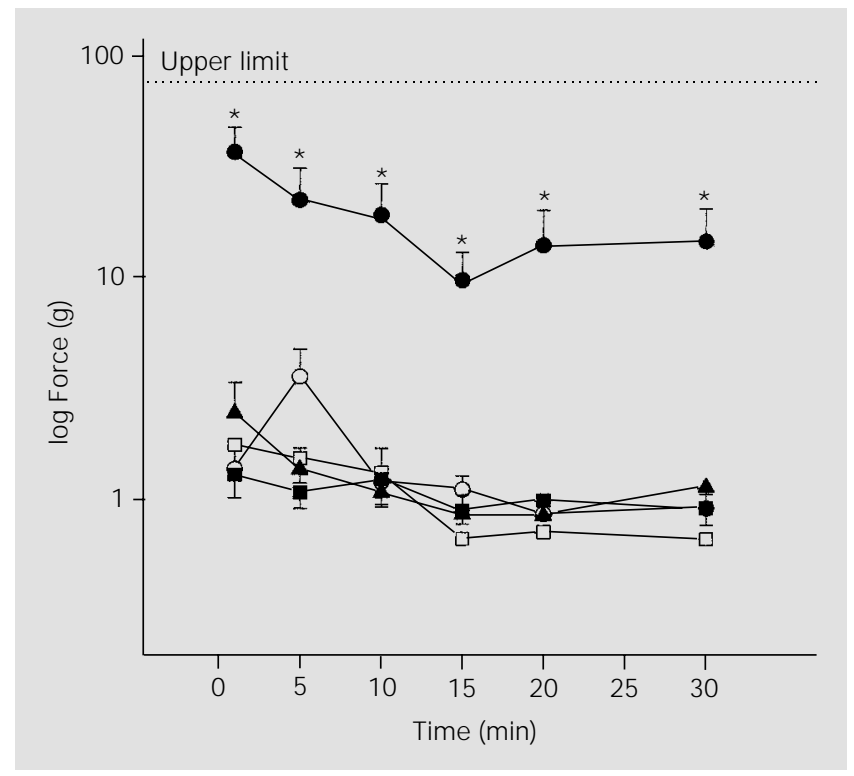

Figure 1 - Time-course of the anti-hyperalgesic effect of electroacupuncture in a model of post-incisional pain in rats. The mechanical hyperalgesia induced by a surgical incision of the plantar surface of the ipsilateral hind paw was evaluated with von Frey filaments. Electroacupuncture was applied over a period of $15 \mathrm{~min}$, beginning $2 \mathrm{~h}$ after surgery, and the mechanical threshold then measured within $1 \mathrm{~min}($ time $=0$ ) and repeated at 5-min intervals for up to $30 \mathrm{~min}$. Control group $(\mathrm{N}=10)$ of nonstimulated rats (open circles); group EA $(N=9)$ of stimulated rats pretreated with saline $(0.1 \mathrm{ml} / \mathrm{kg}$, ip) $10 \mathrm{~min}$ before the stimulation (filled circles); group $\mathrm{S}(\mathrm{N}=8)$ of rats stimulated at non-acupoints (open squares); group S-NAL $(\mathrm{N}=7)$ of non-stimulated rats pretreated with naloxone (1 mg/kg, ip) $10 \mathrm{~min}$ before the stimulation (filled squares), and group EA-NAL $(\mathrm{N}=7)$ of stimulated rats pretreated with naloxone $(1 \mathrm{mg} /$ $\mathrm{kg}$, ip) $10 \mathrm{~min}$ before the stimulation (filled triangles). All animals were lightly anesthetized with halothane during the period of effective or sham electroacupuncture. Points are mean \pm SEM. $* P<0.05$ compared to the control group (Mann-Whitney test). 
dopaminergic systems potentiates the analgesic effect of EA (14). The EA-induced suppression of Fos-like immunoreactivity in structures known to be involved in descending pain mechanisms, such as the locus coeruleus and the ventral portion of the mesencephalic periaqueductal grey, is suggestive that EA can activate descending pain-control mechanisms (15). It was recently demonstrated that post-incisional hyperalgesia can be significantly potentiated in rats in which mesencephalic structures involved in descending pain-control mechanisms were previously blocked with lidocaine (Villareal $\mathrm{CF}$ and Prado WA, unpublished results). Therefore, the model may be useful also for the investigation of the involvement of central pain-control mechanisms in the antinociception induced by EA.

\section{References}

1. Lu GW (1983). Neurobiologic research on acupuncture in China as exemplified by acupuncture analgesia. Anesthesia and Analgesia, 62: 335-340.

2. Cheng SB \& Ding LK (1973). Practical application of acupuncture analgesia. Nature, 242: 559-560.

3. Shu J, Li KY \& Huang DK (1994). The central effect of electroacupuncture analgesia on visceral pain of rats: a study using the [ $\left.{ }^{3} \mathrm{H}\right]$-2-deoxyglucose method. Acupuncture and Electrotherapy Research, 19: 107-117.

4. Leslie A (1974). Acupuncture anesthesia for open heart surgery. American J ournal of Cardiology, 34: 254-255.

5. Matsumoto $\mathrm{T}$, Levy BA \& Perlman $\mathrm{MH}$ (1975). Surgery and acupuncture analgesia. American Surgeon, 41: 422-426.

6. Kho HG, Eijk RJ R, Kapteijns WMMJ \& Van Egmond J (1991). Acupuncture and transcutaneous stimulation analgesia in comparison with moderate-dose fentanyl anaesthesia in major surgery. Anaesthe- sia, 46: 129-135.

7. Bossut DF, Huang ZS, Sun SL \& Mayer DJ (1991). Electroacupuncture in rats: evidence for naloxone and naltrexone potentiation of analgesia. Brain Research, 549: 36-46.

8. Bing Z, Villanueva L \& Le Bars D (1990). Acupuncture and diffuse noxious inhibitory controls: naloxone-reversible depression of activities of trigeminal convergent neurons. Neuroscience, 37: 809-818.

9. Cheng RSS \& Pomeranz B (1979). Electroacupuncture analgesia could be mediated by at least two pain-relieving mechanisms; endorphin and non-endorphin systems. Life Sciences, 25: 1957-1962.

10. Romita W, Suk A \& Henry J L (1997). Parametric studies on electroacupuncture-like stimulation in a rat model: effects of intensity, frequency, and duration of stimulation on evoked antinociception. Brain Research Bulletin, 42: 289-296.

11. Wang B, Tang J, White PF, Naruse R, Sloninsky A, Kariger $\mathrm{R} \&$ Wender $\mathrm{RH}$
(1997). Effect of the intensity of transcutaneous acupoint electrical stimulation on the postoperative analgesic requirement. Anesthesia and Analgesia, 85: 406-413.

12. Brennan TJ , Vandermeulen EP \& Gebhart GF (1996). Characterization of a rat model of incisional pain. Pain, 64: 493-501.

13. Chaplan SR, Bach FW, Pogrel J W, Chung J M \& Yaksh TL (1994). Quantitative assessment of tactile allodynia in the rat paw. J ournal of Neuroscience Methods, 53: 55-63.

14. Han J S \& Trenius L (1982). Neurochemical basis of acupuncture analgesia. Annual Review of Pharmacology and Toxicology, 22: 193-220.

15. Wang H, Li KY, Wu GC \& Cao XD (1995). C-Fos expression in cord and brainstem following noxious stimulation and electroacupuncture plus noxious stimulation. Acupuncture and Electrotherapy Research, 20: 163-174. 